\title{
Immunity against fungi
}

\author{
Michail S. Lionakis, ${ }^{1}$ liyan D. Iliev, ${ }^{2}$ and Tobias M. Hohl ${ }^{3}$ \\ ${ }^{1}$ Fungal Pathogenesis Unit, Laboratory of Clinical Infectious Diseases, National Institute of Allergy and Infectious \\ Diseases, NIH, Bethesda, Maryland, USA. ${ }^{2}$ Jill Roberts Institute for Research in IBD, Department of Medicine, Weill \\ Cornell Medical College, New York, New York, USA. ${ }^{3}$ Infectious Disease Service, Department of Medicine, and Immunology \\ Program, Memorial Sloan Kettering Cancer Center, New York, New York, USA.
}

Pathogenic fungi cause a wide range of syndromes in immune-competent and immunecompromised individuals, with life-threatening disease primarily seen in humans with HIV/AIDS and in patients receiving immunosuppressive therapies for cancer, autoimmunity, and end-organ failure. The discovery that specific primary immune deficiencies manifest with fungal infections and the development of animal models of mucosal and invasive mycoses have facilitated insight into fungus-specific recognition, signaling, effector pathways, and adaptive immune responses. Progress in deciphering the molecular and cellular basis of immunity against fungi is guiding preclinical studies into vaccine and immune reconstitution strategies for vulnerable patient groups. Furthermore, recent work has begun to address the role of endogenous fungal communities in human health and disease. In this review, we summarize a contemporary understanding of protective immunity against fungi.

\section{Introduction}

Humans are exposed to fungi throughout life via inhalation, digestion, and/or traumatic inoculation of fungal particles. The vast majority of these encounters are asymptomatic, and less than 100 of the estimated 5 million fungi species are associated with human disease (1) (Table 1 and Figure 1). Fungi can either exist as spherical yeast cells (e.g., Cryptoccocus neoformans) or as molds that form branching tubular hyphae (e.g., Aspergillus fumigatus). Dimorphic fungi (e.g., Histoplasma capsulatum) grow as molds in the environment and yeasts in human tissue. Candida albicans grows as yeast cells and pseudohyphae, a hyphal form with tapered ends, in human tissue; this morphologic switch is essential for virulence (2).

Fungi were recognized to cause disease during investigations into the scalp dermatophyte infection favus, which was widespread in 19th century Europe (3). German physiologist Robert Remak (1815-1865) immersed favus skin samples in acetic acid and observed fungal hyphae and conidia (named Trichophyton schönleinii in honor of Johann Schönlein, Remak's mentor). In 1842, Remak injected favus crust-isolated material into his forearm and noted growth in the lesions, thereby establishing causality between the fungus and disease.

Several events, including the advent of myeloablative chemotherapy for neoplasia, glucocorticoids and immune modulators for autoimmunity, transplantation for end-organ failure, and the AIDS pandemic, contributed to the emergence of fungal infections in the second half of the 20th century. Novel pathogenic fungi that pose a threat to humans (e.g., Cryptococcus gattii), amphibians (e.g., Batrachochytrium dendrobatidis), and bats (e.g., Pseudogymnoascus destructans) have also been identified (4). In response, research in fungal pathogenesis and antifungal immunity has intensified to inform vaccine- and therapy-based approaches for mycoses (5). This review focuses on insights gained from animal models and patients with primary immune deficiency disorders (PIDDs), but does not cover allergenic or toxinmediated fungal disease $(6,7)$.

Conflict of interest: The authors have declared that no conflict of interest exists.

Published: June 2, 2017

\section{Reference information:}

JCI Insight. 2017;2(11):e93156. https:// doi.org/10.1172/ji.insight.93156.

\section{Antifungal immunity from the bench: contribution of animal models}

This section focuses on antifungal immunity to different yeasts, molds, and dimorphic fungi. Contemporary animal models of fungal infection are reviewed elsewhere (8).

Fungal recognition and immune activation. The fungal cell wall contains polysaccharide and lipid moieties that activate immune responses (9) (Table 2). The cell wall is exterior to the plasma membrane and arranged in layers: the innermost layer typically consists of chitin, an $N$-acetylglucosamine polymer; the adjacent external layer is formed by immunoreactive $\beta-(1,3)$ and $\beta-(1,6)$ glucans, which are concealed by 
many fungi. $H$. capsulatum employs an $\alpha$-glucan layer and the action of a glucanase (9-11). A. fumigatus resting conidia utilize a proteinaceous hydrophobin layer (12), while the hyphal cell wall layer contains galactomannan and galactosaminogalactan, the latter of which conceals inflammatory $\beta$-glucan (13, 14). The $C$. albicans outer cell wall consists of glycoproteins that incorporate $N$ - and $O$-linked mannans and induces inflammatory responses via the mannose receptor and TLR-4 (15). C. albicans mannans conceal $\beta$-glucans as well; the latter are exposed on bud and birth scars during yeast cell division (9). The $C$. neoformans capsule covers the chitinous and $\beta$-glucan-rich cell wall layers and largely consists of glucuronoxylomannan and galactoxylomannan (16). Previously published reviews provide in-depth discussion of fungal cell wall architecture $(9,16-18)$.

At portals of entry fungal cells encounter and bind to antibodies, complement, and soluble pattern recognition receptors. Collectively, these interactions facilitate signaling responses by membranebound receptors and the induction of antifungal effector mechanisms $(5,9)$. In the lung, the collectin pentraxin-3 (PTX3) binds to A. fumigatus conidial galactomannan (19) and facilitates complement

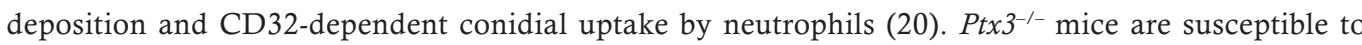
respiratory $A$. fumigatus challenge (19), and a PTX3 polymorphism enhances the vulnerability of hematopoietic cell transplant recipients to invasive aspergillosis (21).

The C-type lectin receptor (CLR) dectin-1 (encoded by Clec7a) binds $\beta$-glucans from a variety of fungi, including those on $C$. albicans bud scars and germinating $A$. fumigatus conidia, and activates signaling responses to Pneumocystis jiroveci, H. capsulatum, Coccidioides posadasii, and Paracoccidioides brasiliensis (5, 9, 22-24) (Figure 2). $\beta$-Glucan binding displaces regulatory phosphatases CD45 and CD148 (25), induces SRC-dependent phosphorylation of the intracellular ITAM-like motif, and recruits the SHP-2 phosphatase (26). SYK docks to this scaffold and transduces signals via PKC- $\delta(27)$ and the VAV family of GEFs (28) to CARD9, which complexes with BCL10 and MALT1 (29) to activate the canonical NF- $\kappa B$ subunits p65 and c-REL (30). Dectin-1 signaling also modulates the noncanonical NF- $\mathrm{B}$ subunit RELB through RAF-1-dependent phosphorylation and deacetylation (31). In macrophages and dendritic cells (DCs), the CARD9/BCL10/MALT1 complex directs $I l 1 b$ transcription and caspase-1- and caspase-8-dependent IL-1 $\beta$ release $(32,33)$, in part via the activity of NRLP3- and AIM2-containing inflammasomes (34). Rubicon can disrupt signal transduction and NF- $\mathrm{BB}$ activation via the CARD9/BCL10/MALT1 complex (35). Dectin-1/SYK/CARD9-dependent cytokines, such as TNF, CXCL2, IL-6, IL-23, and IL-1 $\beta$, promote innate immune activation and Th17 differentiation (36). In addition, dectin-1/SYK signaling in DCs induces IFN- $\beta$ production via IRF5 (37). The role of type I IFN signaling in defense against candidiasis remains controversial, with both protective (37) and detrimental (38) phenotypes reported.

Dectin-1 signaling regulates ERK (also known as MAP kinase) activity via H-RAS and RAS guanine nucleotide-releasing factor 1 (RASGRF1) (39). This pathway regulates macrophage IL-6, IL-1 $\beta$, and TNF, but not IL-12 responses, and is protective during systemic candidiasis (39). c-JUN kinase isoform 1-deficient (JNK1-deficient) mice are resistant to systemic candidiasis (40). Dectin-1-induced JNK1 signaling negatively regulates CD23 (encoded by Fcer2) expression via nuclear factor of activated T cells (NFAT) activation. CD23 binds $\alpha$-mannans and $\beta$-glucans and induces the antifungal effector NOS 2 . Consistent with this model, $C d 23^{-/-}$mice are susceptible to systemic candidiasis (40).

In otherwise nonphagocytic cells, dectin-1 expression promotes phagocytosis of nonopsonized $\beta$-glucan particles $(5,9)$. Bruton's tyrosine kinase (BTK) and VAV-1 interact with dectin-1 in macrophages during $C$. albicans phagocytosis, a process impaired by genetic loss of either protein (41). Dectin-1/SYK/ CARD9 signaling in NADPH oxidase activity is controversial, as dectin-1-dependent (24) and -independent (42) control of $\beta 2$ integrin (CD18) activation and the respiratory burst have been reported in vitro. Murine $\mathrm{Clec}_{7 a^{-/}}$and $\mathrm{Card} 9^{-/-}$neutrophils display no cell-intrinsic defect in killing A. fumigatus conidia, unlike $p 47$ phox $x^{-1}$ neutrophils (43). These data can be reconciled if the major role of dectin-1/CARD9 is to modulate NADPH oxidase and fungal killing via soluble mediators, rather than by cell-intrinsic activation. $\beta 2$ integrins and TLR signaling can collaborate with dectin- 1 to mount macrophage inflammatory responses to $H$. capsulatum and other fungi $(44,45)$.

Dectin-2 (encoded by Clec4n) forms a complex with dectin-3 (encoded by Clec4d) to bind Candida $\alpha$-mannans $(46,47)$ or with mincle (encoded by Clec4e) to bind Malassezia glycolipids (48). Blastomyces dermatiditis, H. capsulatum, $C$. posadasii, and A. fumigatus induce dectin-2 signaling $(43,49)$. As these CLRs lack a signaling domain, heterodimeric complexes signal via the ITAM-coupled adaptor FcR $\gamma(9)$. 
Table 1. Common human fungal diseases and associated PIDDs

\begin{tabular}{|c|c|c|c|}
\hline $\begin{array}{l}\text { Mycosis } \\
\text { Fungal species } \\
\text { Most common }\end{array}$ & Morphotype & Clinical Syndromes & $\begin{array}{l}\text { Genes (syndromes) linked to enhanced } \\
\text { susceptibility }{ }^{A}\end{array}$ \\
\hline
\end{tabular}

Aspergillosis Mold Pneumonia and systemic disease in immune-compromised NADPH oxidase (CGD); STAT3 (HIES);

Aspergillus spp., A. hosts; allergenic disease (e.g., ABPA) in atopic hosts; cavitary CARD9 (extrapulmonary aspergillosis);

disease in setting of structural lung disease; deep tissue GATA2 (MonoMAC syndrome); CD18

fumigatus, A. flavus, A. niger, organ disease (e.g., brain abscess, osteomyelitis) in CGD and

(LAD); ELA2, HAX1 (SCN)

A. terreus, $A$. nidulans (in CARD9-deficient patients

Mucormycosis
Rhizopus, Mucor and $\quad \begin{gathered}\text { Pneumonia, sinus, and systemic disease in immune- } \\ \text { Rhizompromised hosts and in diabetic ketoacidosis }\end{gathered}$

Rhizomucorspp.

Fusariosis

Mold Pneumonia, cutaneous, bloodstream, and systemic disease in immune-compromised hosts; fungal keratitis

NADPH oxidase (CGD), STAT1 GOF

Fusarium oxysporum and $F$. immune-compromised hosts; fungal keratitis mutations

solani

Dermatophytosis

Mold

Skin and nail infections (i.e., keratinized tissues), e.g., tinea pedis (athlete's foot), ringworm

NADPH oxidase (CGD); CARD9 (deep

Epidermophyton, tissue infections)

spp.

\section{Sporotrichosis \\ Sporothrix schenkii \\ Blastomycosis}

Blastomyces dermatiditis

$\begin{array}{lc}\text { Histoplasmosis } & \text { Dimorph } \\ \text { H. capsulatum (North } & \text { (intracellular in } \\ \text { America), H. dubousii (Africa) } & \text { macrophages) }\end{array}$

Lymphocutaneous disease, ascending lymphangitis

Pneumonia; skin, mucosal, skeletal, and genitourinary disease

Pneumonia, mediastinal granuloma and fibrosis, disseminated histoplasmosis

\section{Coccidioidomycosis}

C. immitis, C. posadasii

Paracoccidioidomycosis

P. brasiliensis

Penicilliosis

Talaromyces marneffei

Pneumocystosis

P. jiroveci

\section{Dimorph \\ Pneumonia, skeletal disease, meningitis}

Dimorph

Dimorph

Trophozoites

(asexual) and

cysts (sexual)

\section{Candidiasis}

C. albicans, C. tropicalis

C. glabrata, C. krusei,

C. parapsilosis, C. auris

\section{Dimorph Thrush, vulvovaginitis; bloodstream and systemic infections}

Yeast in immune-compromised patients; AIDS-defining illness; deep tissue single organ disease (e.g., kidney or brain abscess, osteomyelitis) in CCD and other PIDDs; deep tissue disease uncommon in syndromes associated with CMC with the exception of CARD9 deficiency

Cryptococcosis
C. neoformans, C. gattii
Chromoblastomycosis
Fonsecaea pedrosii, and
others
Eumycetoma
Scedosporium spp., Madurella
spp., and others

Yeast
(pigmented,
encapsulated)
Yeast
(pigmented)

Molds (pale or Chronic infection of cutaneous and subcutaneous tissues;

Chronic infection of cutaneous and subcutaneous tissues
Pneumonia; skin, mucosal, and skeletal disease

Pulmonary, skin, mucosal, and disseminated disease

Pneumonia; common AIDS-defining illness

CD40 ligand, $>30$ genes that underlie SCID; MHC class II deficiency; NEMO; DOCK8 (HIES); CARD11; WiskottAldrich syndrome

CMC: IL17F; IL17RA; IL17RC; ACT1; STK4; IRF8; CARD9; STAT1 GOF mutations; STAT3 (HIES); RORC; AIRE; anti-IL-17 autoantibodies (thymoma) CNS disease: CARD9

Invasive and deep tissue disease: ELA2, HAX1 (SCN), CCD, complete MPO deficiency

Anti-CM-CSF or -IFN- $\gamma$ antibodies; CD40 ligand; CATA2; IL12R; STAT3

(HIES)
Pneumonia, meningitis; AIDS-defining illness

\author{
IL12RB1; IFNGR1; STAT1 GOF \\ MAGT1, RAG1 (idiopathic CD4 \\ lymphopenia); DOCK8 (HIES); CD40 \\ ligand \\ IL12RB1; IFNGR1; STAT1 GOF
} IL12RB1

${ }^{A}$ We did not discuss all listed gene defects associated with mycoses in the text. The reader is referred to references 64 and 65 for a more comprehensive discussion and additional links to the primary literature. ABPA, allergic bronchopulmonary aspergillosis; CARD9, caspase recruitment domain-containing protein 9; CMC, chronic mucocutaneous candidiasis; CCD, chronic granulomatous disease; CNS, central nervous system; DOCK8, dedicator of cytokinesis 8; GM-CSF, granulocyte-macrophage colony-stimulating factor; GATA2, GATA-binding protein 2; GOF, gain of function; HIES; hyper-IgE syndrome; LAD; leukocyte adhesion deficiency; NEMO, NF-KB essential modulator; PIDDs, primary immune deficiency disorders; SCID; severe combined immunodeficiency; SCN; severe combined neutropenia; STAT, signal transducer and activator of transcription. 


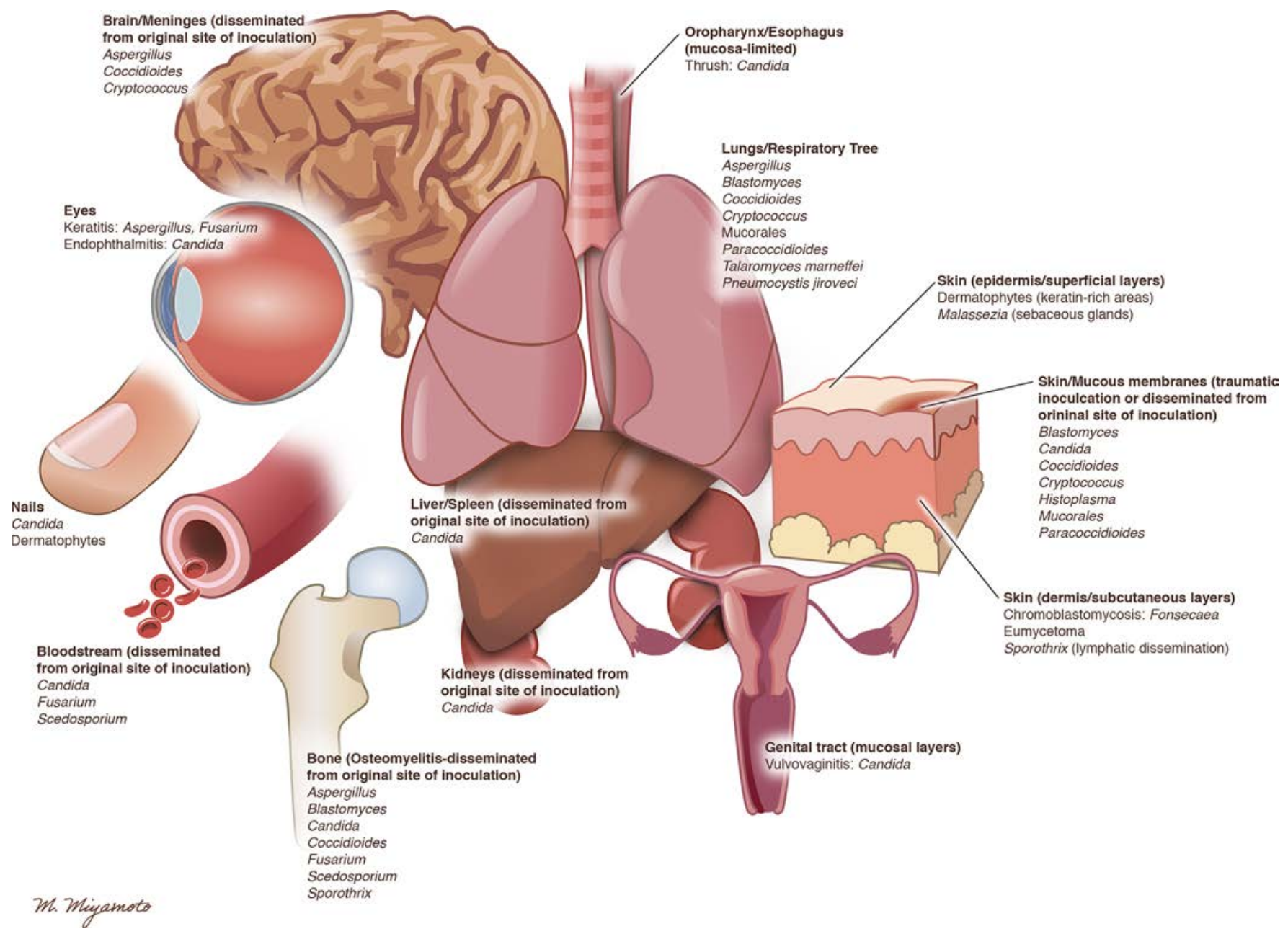

Figure 1. Human fungal diseases. The figure depicts the anatomic sites that are most commonly affected by the fungal genera listed below each organ system. At several sites, fungal disease occurs due to inoculation at the site. For example, humans inhale infectious conidia or desiccated fungal cells of all genera listed under lungs and respiratory tree. For other sites, such as the liver, spleen, and blood stream, disease is the result of dissemination of the indicated fungus from the initial inoculation site. Illustrated by Mao Miyamoto.

Following fungal recognition, the E3 ubiquitin ligase CBLB ubiquitinates dectin-1, dectin-2, and SYK, targeting them for degradation (50-52). In systemic candidiasis, $\mathrm{Cblb}^{-/-}$mice exhibit reduced renal fungal burden and improved survival due to decreased inflammation-driven tissue damage (50-52). In contrast, TRIM62-mediated CARD9 ubiquitination is essential for BCL10 interactions, NF-kB activation, and defense against candidiasis (53).

Mincle/SYK/CARD9 signaling mediates responses to Fonsecaea pedrosii, an agent of chromoblastomycosis (54). Mincle-induced cytokines are insufficient to clear this soft tissue infection, and application of TLR-7 agonist imiquinod to fungal lesions can restore sterilizing immunity (55). In DCs, mincle signaling activates the E3 ubiquitin ligase MDM2, in turn suppressing dectin-1- and IRF1-dependent Il12a transcription (56). This MDM2-dependent pathway impairs Th1 responses and may contribute to the chronicity of chromoblastomycosis. During murine pneumocystosis and candidiasis, mincle signaling enhances fungal clearance but is dispensable for survival $(57,58)$.

Loss of individual CLRs results in variable susceptibility to C. albicans $(22,23)$ and $A$. fumigatus $(24$, 43), in part due to strain-specific differences in CLR activation, as shown for C. albicans (59). Consistent with SYK/CARD9 being central for CLR signal integration, mice with hematopoietic or DC-specific Syk deletion or with global CARD9 deficiency are highly susceptible to $C$. albicans $(29,60)$ and $A$. fumigatus (43) challenge. CARD9/SYK-dependent susceptibility maps to defective cytokine responses that control neutrophil, NK, and T cell trafficking or activation. During systemic candidiasis, DCs secrete IL-23 in a SYK-dependent manner, prompting IL-17A-dependent (hereafter referred to as IL-17) NK 
Table 2. Mammalian signaling receptors and cognate fungal ligands and species

\begin{tabular}{|c|c|c|c|}
\hline C-type lectin receptors: & Fungal ligand & Fungal species ${ }^{A}$ & References $^{B}$ \\
\hline Dectin-1 (Clec7a) & $\beta$-Glucans & $A F, C A, C P, E R, H C, P B, P C$ & $(23,24,44,49,59,184)$ \\
\hline Dectin-2 (Clec4n) & $\alpha$-Mannans, 0 -linked mannoproteins & AF, BD, CA, CP, CG & $(48,49,97,185)$ \\
\hline Dectin-3 (CLECSF8, MCL, Clec4d) ${ }^{\mathrm{C}}$ & $\alpha-M a n n a n s$ & CA & $(47,186)$ \\
\hline Mincle (Clec4e) & $\alpha$-Mannosyl residues, glyceroglycolipids & CA, FP, MS, PC & $(48,54,58)$ \\
\hline CD209 (DC-SIGN) & Galactomannan, mannans & $A F, C A$ & (187) \\
\hline Mannose Receptor & $\mathrm{N}$-linked mannans, mannans & $C A, P C$ & $(15,188)$ \\
\hline TLR-1 and TLR-2 & Glucuronoxylomannans & CN & (190) \\
\hline TLR-2-TLR-6 & Phospholipomannans, glucuronoxylomannans & $\begin{array}{l}\text { CA } \\
\text { CN }\end{array}$ & $(191)$ \\
\hline TLR-4 & O-linked mannans, rhamnomannans & $\mathrm{CA}, \mathrm{SA}$ & $(15,189,192)$ \\
\hline TLR-9 & Unmethylated DNA & $A F, C A$ & (63) \\
\hline NLRP3 & Unknown & $A F, C A$ & $(34,196)$ \\
\hline \multicolumn{4}{|l|}{ Other receptors: } \\
\hline CD14 & $\alpha$-(1,4)-Glucans & SA & (197) \\
\hline CD23 (Fcer2a) & $\alpha$-Mannan, $\beta$-glucan & CA & $(40)$ \\
\hline CD36 & & CN, CA & (198) \\
\hline CR3 (integrin $\alpha_{2} / \beta_{\mathrm{M}} ;$ CD11b/CD18; Mac-1) & $\beta$-Glucan & $\mathrm{AF}, \mathrm{CA}, \mathrm{HC}$ & $(42,44,45,72,73)$ \\
\hline Galectin-3 & $\beta$-Mannosides & CA & (199) \\
\hline Lactosylceramide (glycosphingolipid) & & $P C$ & $(200)$ \\
\hline Pentraxin-3 (soluble) & Galactomannan & AF & $(19,62)$ \\
\hline \multicolumn{4}{|c|}{ 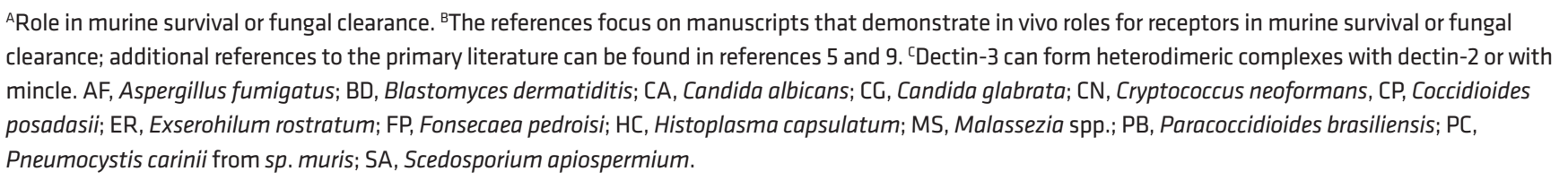 } \\
\hline
\end{tabular}

cell release of GM-CSF, thereby activating candidacidal neutrophils at infection sites $(60,61)$.

CLR-independent pathways also mediate fungus-induced inflammation. Fungal ligands activate TLR1-4, TLR-6, TLR-9, and NOD2 signaling in a variety of cell types $(5,9)$. For example, PTX3-opsonized $A$. fumigatus conidia activate TLR-4/MD-2/TRIF-dependent signaling that mediates IL-10 production (62). Following internalization, A. fumigatus conidia trigger macrophage TLR-9-, calcineurin-, and BTK-dependent TNF release (63); however, humans with NLR or TLR/MyD88 signaling defects do not manifest with fungal infections $(64,65)$. A detailed discussion of Mendelian defects that do manifest with fungal infections follows later in this review.

Fungal killing. Neutrophils rapidly internalize conidia and/or yeast cells and direct the production of ROS to the fungal phagosome (66). In macrophages, NADPH oxidase-dependent LC3-associated phagocytosis represents a potential clearance mechanism for $A$. fumigatus conidia $(67,68)$. Nutritional immunity, exemplified by zinc and iron sequestration, is critical for macrophage defense against intracellular $H$. capsulatum yeast cells (69), against fungal keratitis (70), and in chronic granulomatous disease (CGD) patients with defects in NADPH oxidase (71-73).

Fungal hyphae (A. fumigatus) and pseudohyphae ( $C$. albicans) induce NADPH oxidase-dependent neutrophil extracellular trap (NET) formation (74), a form of neutrophil programmed cell death termed NETosis. NETs contain extracellular nucleic acids, histones, and granular proteins, including calprotectin and PTX3 $(75,76)$, and ensnare fungal organisms that are too large for phagolysosomal killing (74). Neutrophil calprotectin, a major NET component, is important for antihyphal host defense during murine $A$. fumigatus keratitis yet dispensable for anticonidial defense following respiratory $A$. fumigatus challenge (77), illustrating a fungal 


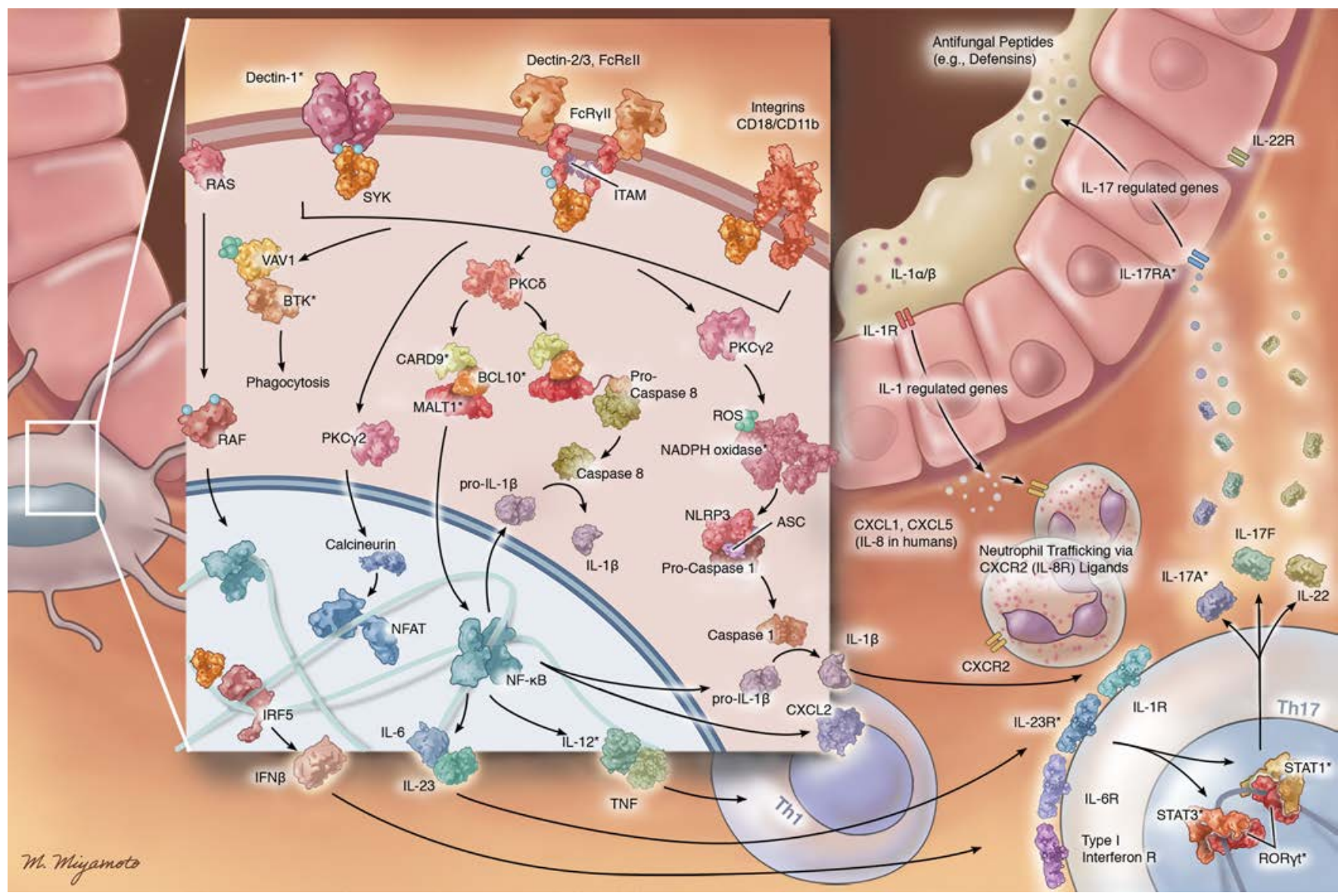

Figure 2. Model of fungus-induced CLR signaling in antifungal defense. At the site of inoculation, particulate fungal polysaccharides bind C-type lectin receptors (CLRs) and Fc receptors (FcRs), resulting in SYK activation via ITAM signaling in the receptor tail, the FcR $\gamma$ signaling adaptor, or integrin receptor activation. The ensuing PKC $\delta$ and CARD9 activation is critical for caspase- 1 and caspase- 8 activity, MAP kinase signaling (not shown), NF-KB activation, and cytokine production. SYK-dependent PLC $\gamma 2$ activation is linked to NADPH oxidase assembly and calcineurin-dependent NFAT activation. Dectin/SYK signaling controls IRF5-dependent IFN- $\gamma$ production. VAV1 and BTK can interact with dectin-1 to mediate phagocytosis of Candida albicans in macrophages. BTK may also promote calcineurin activation and the production of NFAT-regulated cytokines. The model depicts fungal killing in the phagosome. Myeloid cellderived cytokines promote the differentiation of both Th1 cells and Th17 cells. Th17 and innately derived IL-17 activate epithelial responses that coordinate clearance of fungal cells at mucosal surfaces. The asterisks indicate genetic or humoral defects that predispose humans to a spectrum of fungal disease. Myeloid and epithelial cells collaborate to regulate CXCR2-dependent neutrophil recruitment to portals of infection. Illustrated by Mao Miyamoto.

morphotype-specific role in host defense. A. fumigatus hydrophobins and galactosaminogalactan enhance fungal resistance to NETs $(78,79)$. The contribution of NETs to fungal clearance in vivo remains difficult to quantify, because researchers lack experimental tools that specifically disrupt NETosis in murine models.

Mucosal defense and fungal tissue invasion. At mucosal surfaces, IL-17 is critical for antifungal immunity. Innate lymphoid, Th17, and $\gamma \delta \mathrm{T}$ cells produce IL-17 following $C$. albicans challenge in the oropharynx or skin $(80,81)$. Skin sensory neurons participate in $C$. albicans detection and activate dermal DCs via the neuropeptide calcitonin-related gene product to direct IL-23-dependent $\gamma \delta$ T cell IL-17 release (82). Oral epithelial cells represent an important IL-17 signaling target, as mice lacking IL-17-sensing capacity in these cells display a phenotype similar to that of $I l 17 \mathrm{ra}^{-/-}$mice (83). The IL-17-dependent transcriptional response of oral epithelial cells includes antimicrobial peptides, including $\beta$-defensin 3 (DEFB3). Accordingly, Defb $3^{-/-}$mice are susceptible to oropharyngeal candidiasis (83). In C. albicans-infected kidneys, IL-17dependent responses include activation of the kallikrein/kinin system, which prevents apoptosis of tubular cells (84). Neutrophil recruitment to infected oral tissues is complex, with reports of IL-17-dependent and -independent trafficking pathways $(85,86)$. One IL- 17 -independent mechanism involves IL- $1 \alpha / \beta-$ sensing oral keratinocytes that regulate neutrophil influx via CXC-chemokine release and indirect control over G-CSF-dependent granulopoiesis (87). Thus, mucosal infections induce crosstalk between epithelial and hematopoietic cells to regulate innate activation and fungal clearance. 
Fungal invasion of epithelial and endothelial cells contributes to tissue damage and disease dissemination. During mucormycosis, the endothelial receptor glucose-regulated protein 78 (GRP78) enables Rhizopus oryzae hyphae binding to endothelial cells (88) via spore coat protein surface proteins (CotH), primarily CotH3. Metabolic changes associated with hyperglycemia and diabetic ketoacidosis (DKA) enhance endothelial GRP78 expression, resulting in GRP78-dependent fungal tissue invasion (89). The widespread expression of CotH family members among Mucorales and absence from other fungal pathogens may explain the unique susceptibility of patients with DKA to mucormycosis. Consistent with this model, antibodies directed against GRP78 or CotH3 protect mice from mucormycosis (90).

The $C$. albicans adhesin Als3 mediates binding to biotic and abiotic surfaces, including epithelial and endothelial cells, and triggers internalization via E- and N-cadherin (91). The adhesin Hwp1 and the heat-shock protein Ssa1 also contribute to this process (92). An Als3p-based vaccine has emerged as a promising candidate to prevent mucosal and systemic disease (93) (NIH ClinicalTrials.gov identifiers: NCT01926028, NCT02996448). Following epithelial attachment, $C$. albicans pseudohyphae secrete the pore-forming, cytolytic peptide toxin candidalysin that is essential for virulence (94). At the onset of epithelial invasion, candidalysin activates MAPK signaling and c-FOS activation at sublytic concentrations (94). Epithelial activation leads to IL-1 $\alpha$, IL-6, G-CSF, and GM-CSF release, alerting host cells to the presence of invasive pseudohyphae.

A. fumigatus conidia express the lung mucin-binding lectin FleA that promotes lung macrophages to internalize conidia (95). Mice challenged with $\Delta$ fleA conidia have more severe pneumonia than animals challenged with WT conidia. Thus, FleA represents an essential target of the immune system to clear inhaled conidia. A. fumigatus hyphae also invade epithelial and endothelial cells, a property mediated in part by the CalA protein (96) and by galactosaminogalactan (14). Although CalA is dispensable for host cell adherence, CalA stimulates integrin $\alpha 5 \beta 1$-dependent hyphal endocytosis and is required for virulence in immunosuppressed mice (96).

Trained immunity and fungal infections. Humans maintain sterilizing antifungal immunity in the lung despite daily inhalation of thousands of fungal cells; therefore, single-inoculum animal models are limited in revealing immunologic responses that arise in response to multiple challenges. Repeated exposure to $A$. fumigatus antigens gives rise to ROR $\gamma \mathrm{t}^{+} \mathrm{IL}-17^{+}$neutrophils in response to IL-6 and IL-23 production (97). IL-17+ neutrophils express IL-17RC and signal in a paracrine manner to boost fungicidal activity following secondary challenge in the eye and lung $(97,98)$.

Murine Ly6 $C^{\text {hi }}$ monocytes represent effector cells against $C$. albicans (99), A. fumigatus (100), and $B$. dermatiditis (101). During repetitive challenges with $\beta$-glucans, murine Ly6Chi monocytes and human $\mathrm{CD} 14^{+}$monocytes exhibit attributes reminiscent of immunologic memory, including enhanced responsiveness, based on cytokine responses (102). $\beta$-Glucan priming induces epigenetic and metabolic changes in monocytes, the latter of which associates with a shift from oxidative phosphorylation to glycolysis via a dectin-1/AKT/mTOR/HIF-1 $\alpha$ signaling pathway (103) and increased glutaminolysis (104). In macrophages, $\beta$-glucan priming partially reverses LPS exposure-associated chromatin modifications, specifically the silencing of proinflammatory genes (105). In humans, a recent study proposes a role for STAT1 signaling in eliciting trained immunity, and by extension, in protection against chronic mucocutaneous candidiasis (CMC) (106)

Adaptive antifungal immunity. Antibody-mediated immunity to fungal pathogens has been extensively reported in the literature (107). For example, natural fungal polysaccharide-targeting IgM antibodies enhance DC-mediated recognition of fungal antigen, the development of Th2 and Th17 responses, and $\mathrm{B}$ cell isotype class-switch recombination during murine pneumocystosis (108). In a model of X-linked agammaglobulinemia, pulmonary and CNS cryptococcal disease progressed rapidly, in part due to IgM-dependent defects in macrophage phagocytosis (109). Although there is significant support for the concept that antibody-dependent opsonization and complement activation enhance fungal clearance in animal models (107), loss of antibody-mediated immunity is generally compensated by alternate effector systems, such as myeloid and $\mathrm{CD}^{+} \mathrm{T}$ cell-mediated immunity. Additionally, humans with humoral immune defects are generally not susceptible to fungal disease; however, protective fungus-specific antibodies are being researched as an adjunctive therapy in preclinical models (107). Several groups have generated protective antibodies that form the basis for vaccine strategies in murine models of fungal disease and in a phase I trial for recurrent vulvovaginal candidiasis (110-113) (NIH ClinicalTrials.gov identifier: NCT01067131). 
In the 1980s and 1990s, the dominant CD4+ $\mathrm{T}$ cell-dependent protection model involved the dichotomous differentiation of $\mathrm{CD}^{+} \mathrm{T}$ cells into Th1 and Th2 subsets. More recent studies have shown that antibody- or cytokine-mediated disruption of Th1 immunity or Th2-favoring interventions correlate with adverse outcomes in murine fungal infection models $(5,9)$. The discovery of Th17 and regulatory $\mathrm{T}$ cells (Tregs), the development of $\mathrm{CD}^{+} \mathrm{T}$ cell receptor-transgenic mice (114-116), the identification of fungal epitopes that elicit antigen-specific responses (117-119), and the precise phenotyping of PIDD patients with mycoses have advanced insight into $\mathrm{CD} 4^{+} \mathrm{T}$ cell-driven antifungal immunity (120).

At the site of infection, fungal cells and antigens are internalized by tissue-resident DC subsets and monocyte-derived cells (Mo-DCs). In the lung, Ly6 $\mathrm{C}^{\text {hi }}$ monocytes and derivative Mo-DCs transport $A$. fumigatus conidia and $B$. dermatiditis yeast cells to lung-draining lymph nodes $(121,122)$. In a $B$. dermatiditis vaccine model, fungal protease-dependent cleavage of CCR2 ligands impedes lung Ly6 $\mathrm{C}^{\text {hi }}$ monocyte trafficking and the development of vaccine protection (123). However, Mo-DCs are not required for direct $\mathrm{CD}^{+} \mathrm{T}$ cell priming due to antigen transfer to lymph node-resident DCs (122). Mo-DCs, as well as IRF4-

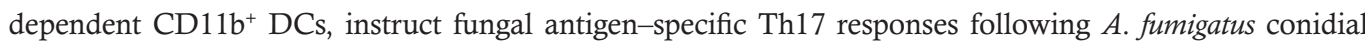
challenge $(124,125)$. In the oral mucosa, CCR7-dependent transport of candidal antigens to draining lymph nodes results in antigen presentation by FLT3L-dependent migratory DCs and by Mo-DCs, both of which have the capacity to prime antigen-specific $\mathrm{CD} 4^{+} \mathrm{T}$ cells (116).

In the skin, epidermal Langerhans cells recognize $C$. albicans yeast cell $\beta$-glucan via dectin- 1 , release IL-6, and instruct Th17 cell differentiation. These Th17 cells protect against secondary skin infection, but not against secondary systemic challenge (126). When $C$. albicans pseudophyphae penetrate the epidermis, dermal CD11b ${ }^{-} \mathrm{CD} 103^{+}$DCs drive differentiation of Th1 cells (126), which protect against secondary systemic challenge, but not secondary skin challenge (126). Thus, the cellular requirements for $\mathrm{CD}^{+} \mathrm{T}$ cell priming and differentiation vary by anatomic site and fungal morphology, and, in most cases, involve antigen transfer from migratory DCs and Mo-DCs to lymph node-resident DCs.

During pulmonary $A$. fumigatus challenge, T helper cell differentiation occurs incrementally. Expression of the transcription factor T-bet, which controls Th1 differentiation, is detectable in antigen-specific CD4 ${ }^{+} \mathrm{T}$ cells in lung-draining lymph nodes and is enhanced by MyD88-dependent signals. In infected airways, MyD88independent signals promote further Th1 differentiation and IFN- $\gamma$ production (114). In this model, dectin-1 signaling counters Th1 differentiation by limiting innate IL-12p35 and IFN- $\gamma$ production (124). Vaccine immunity to dimorphic fungi relies primarily on pulmonary Th17 cells that likely facilitate mononuclear phagocyteand neutrophil-dependent killing of these pathogens $(127,128)$. Recently, researchers identified a fungal calnexin epitope that is widely conserved among ascomycetes (118). Vaccine delivery of fungal calnexin elicited calnexin-specific $\mathrm{CD} 4^{+} \mathrm{T}$ cells that conferred protection against the three North American dimorphic fungal pathogens (118). Calnexin represents the most promising candidate for vaccine design against these mycoses.

Protective Th17 responses at mucosal surfaces can be enhanced by Treg-mediated IL-2 consumption, which enhances IL-17 and IL-22 release by responding Th17 cells and increases resistance to mucosal fungal infection (129). Despite the obligate requirement for IL-17 signaling during systemic candidiasis (61), FOXP3 ${ }^{+}$ Treg-mediated potentiation of Th17 responses appears detrimental (130), consistent with the notion that high IL-17 levels lead to immunopathology, as described for type I IFN (38) and CCR1 signaling (131).

During pulmonary cryptococcosis, mammalian chitotriosidase digests fungal chitin, thereby stimulating lung-resident $\mathrm{CD}_{11} \mathrm{~b}^{+} \mathrm{IRF} 4^{+} \mathrm{DCs}$, which in turn promote a Th2-dominant response to virulent serotype A strains (119). Compared with WT mice, $C$. neoformans challenge in chitotriosidase-deficient animals elicited dramatically lower antigen-specific Th2 cell numbers and extended murine survival, despite similar lung fungal burden (119). These data support a model in which antigen-specific Th2 cells exacerbate cryptococcosis by augmenting tissue damage without effecting fungal growth. Consistent with these findings, chitosan-deficient (i.e., deacetylated chitin) cryptococcal strains elicit cytokine mediators that promote a Th1-biased response (132). Similarly, the chitin content of individual A. fumigatus strains correlates with the magnitude of Th2 responses and lung eosinophil recruitment (133). The role of eosinophils in fungal clearance remains poorly understood, with several recent reports suggesting these cells help mediate fungal clearance (134-136).

\section{Antifungal immunity: lessons from fungal disease-associated Mendelian disorders}

In the 1970s-1980s, characterization of two prototypic PIDDs that result from phagocyte oxidative machinery defects - CGD, caused by mutations in NADPH oxidase subunits and myeloperoxidase (MPO) deficiency 
$(137,138)$ - catalyzed our recognition that phagocyte oxidative cytotoxicity is critical for protection against invasive fungal disease in humans. The spectrum of clinical phenotypes of these disorders highlights the fungus-specific dependence on oxidative versus nonoxidative cytotoxic mechanisms for defense. For example, pulmonary aspergillosis is the signature fungal infection in CGD (137). Yet, only $\sim 40 \%$ of CGD patients develop aspergillosis during their lifetime, despite daily inhalation of ubiquitous conidia. This observation indicates that nonoxidative mechanisms compensate in the remainder of the Aspergillus-exposed patients; better understanding of these mechanisms is an important future research direction $(9,69,71,77,139)$. Invasive infections by other molds, such as Mucorales and Fusarium, or by commensal Candida yeasts are very uncommon ( $<5 \%)$, whereas invasive infections by endemic dimorphic fungi and Cryptococcus, or CMC do not develop in CGD $(137,140)$. In contrast, MPO deficiency is not permissive to infections by inhaled molds, and only a minority $(<5 \%)$ of MPO-deficient patients develops invasive candidiasis. These clinical observations show that human phagocytes differentially depend on their oxidative capacity to effectively control different fungal pathogens. Importantly, while both CGD and MPO-deficient patients manifest defective hypochlorous acid production within phagocytes $(137,138)$, they exhibit distinct features in the phenotypic expression of fungal disease. The discrepancy in susceptibility to mold infection in these patients may reflect, at least in part, a potentially significant contribution of superoxide anion-dependent $\mathrm{K}^{+}$influx in activating phagolysosomal granule proteases (141), which is defective with NAPDH oxidase, but not MPO, deficiency.

In the 1990s-2000s, the discovery of PIDDs resulting from alterations of IL-12/IFN- $\gamma$ signaling uncovered the critical role of IL-12/IFN- $\gamma$-dependent lymphocyte/macrophage crosstalk in control of intracellular pathogens, including endemic dimorphic fungi, Cryptococcus, mycobacteria, and Salmonella (65). This pathway is dispensable for control of inhaled molds and mucosal fungal infection in humans. Some of these disorders respond clinically to mechanism-based immunotherapy with IFN- $\gamma$ or IFN- $\alpha$ (142). Similarly, neutralizing IFN- $\gamma$ and GM-CSF autoantibodies can underlie adult-onset acquired immunodeficiency characterized by endemic dimorphic fungal (143) and CNS cryptococcal disease (144). Patients with alveolar proteinosis due to impaired GM-CSF signaling develop aspergillosis (145), consistent with murine studies (146).

During the last decade, careful clinical phenotyping combined with next-generation sequencing technologies revealed the critical balance of JAK/STAT signaling in human antifungal immunity (147). Patients with STAT1 gain-of-function (GOF) mutations and those with hyper-IgE syndrome (Job's syndrome) due to STAT3 loss-of-function (LOF) mutations develop fungal disease with overlapping and distinct features. STAT1 GOF mutations lead to disseminated infections by intracellular dimorphic fungi, CMC, and/or infections by inhaled molds in the absence of structural lung disease $(65,148)$. STAT3 LOF mutations also result in CMC, but pulmonary infections by inhaled molds occur as a consequence of structural lung disease caused by prior bacterial lung infections. In this cohort, infections by intracellular dimorphic fungi occur very rarely and, when they do, tend to involve the gastrointestinal tract (149). In contrast, LOF STAT1 mutations or GOF STAT3 mutations do not cause fungal disease (65).

Mutations in the transcription factor GATA2 result in a protean PIDD $(65,150)$ that brings together (a) susceptibility to bacterial, fungal and viral disease, (b) malignancy, and (c) vascular abnormalities. From a fungal disease standpoint, GATA2 haploinsufficiency leads to infections by endemic dimorphic fungi, Cryptococcus, and Aspergillus, but not Candida. GATA2-deficient patients exhibit monocytopenia that, consistent with recent murine models, likely contributes to fungal susceptibility $(100,150)$.

The discovery of kindreds with CMC that carry mutations in IL17F, IL17RA,IL17RC, and NF- $\mathrm{BB}$ activator 1 ( $A C T 1)$, and complementary studies in IL-17-mutant mice discussed above indicate that IL-17 signaling is indispensable for mucosal, but not systemic, antifungal immunity $(64,65)$. Patients with $I L 17 F$ or IL17RC mutations only develop CMC, whereas those with IL17RA or ACT1 mutations also manifest cutaneous staphylococcal and/or pulmonary bacterial infections, indicating that IL-17E/IL-25 signaling may specifically map to bacterial defense at mucosal surfaces $(151,152)$. CMC susceptibility has also been linked to mutations in other genes $(64,65)$, most of which either directly or indirectly interfere with Th17 development and/or responses (Figure 2). These include AIRE deficiency in autoimmune polyendocrinopathy-candidiasis-ectodermal dystrophy (APECED) (153), Job's syndrome in which STAT3 regulates ROR $\gamma \mathrm{t}$-dependent Th17 development, the various genetic forms of severe combined immunodeficiency disorder (154), and mutations in STAT1, RORC, STK4, IRF8, DOCK8, IKBA, CLEC7A, and CARD9 (64, 65, 155). A $C L E C 7 A$ polymorphism is also associated with susceptibility to invasive aspergillosis in hematopoietic cell transplant recipients (156). 
CARD9 deficiency exhibits three distinctive features among Mendelian disorders of antifungal immunity (157). First, a substantial proportion of CARD9-deficient patients exhibit adult-onset fungal disease, in contrast to early childhood onset typical of other PIDDs. It is unclear whether a different threshold of fungal exposure and/or additional genetic, environmental, or other factors accounts for this observation. Second, CARD9 deficiency is the only PIDD in which both mucosal and systemic fungal disease develop, and susceptibility is restricted to fungi without concurrent bacterial or viral infections. Invasive candidiasis, CMC, invasive phaeohyphomycosis, and superficial and deep-seated dermatophytosis have also been reported (157). In contrast to CARD9, MALT1 or BCL-10 deficiency results in a narrower spectrum of fungal susceptibility, with only mucosal infections reported to date (157). Third, CARD9 deficiency strikingly results in infections at anatomical sites that are not typically associated with the specific pathogens. For example, CARD9-deficient patients exhibit a predilection for Candida meningoencephalitis, without disease in kidney, liver, or spleen, all of which are commonly affected in CARD9-sufficient patients $(157,158)$. CARD9 is critical for neutrophil recruitment to the Candidainfected CNS but not for neutrophil recruitment to the Candida-infected kidney or Staphylococcus-infected CNS (159). This tropism is mediated by CARD9-dependent production of CXC chemokines by resident glial cells and neutrophils in the Candida-infected CNS, while neutrophil-intrinsic chemotaxis and effector functions are largely intact in CARD9-deficient humans and mice (159-161). Development of CNS and intra-abdominal aspergillosis is another example of altered tissue tropism in CARD9-deficient patients (162) and results from defective CARD9-dependent neutrophil chemoattractant production in and neutrophil mobilization to extrapulmonary infection sites. The absence of pulmonary Aspergillus involvement in CARD9 deficiency may be explained by compensatory IL-1R/MyD88-dependent neutrophil lung recruitment $(161,163)$. Collectively, these human observations have led to our appreciation that CARD9 appears to mediate fungus-, cell type-, and organ-specific neutrophil recruitment during invasive fungal infection.

GM-CSF and G-CSF were reported to improve the outcome of a small number of CARD9-hypomorphic patients with CNS candidiasis (164). In this cohort, mutant CARD9 was impaired in its ability to complex with RASGRF1, but not with BCL10 and MALT1 (165). The mechanism by which these cytokines bypass the CARD9-dependent immune defect in this cohort and the generalizability of this protection in other CARD9-deficient patients remain to be elucidated.

\section{Antifungal immunity and the study of the mycobiome}

While commensal fungi have been noted in humans and mice for over five decades, lack of culture-independent methods, delay in developing high-throughput rDNA sequencing methods, and paucity of annotated reference databases to classify fungal rDNA amplicons delayed characterization of endogenous fungal communities, termed the mycobiota $(166,167)$. Although fungi comprise less than $1 \%$ of total microbial rDNA sequences at different anatomic sites, the size of fungal cells $(\sim 3-10 \mu \mathrm{m}$ diameter for most yeast cells, $2-5 \mu \mathrm{m}$ diameter for typical mold conidia, and 10 to hundreds $\mu \mathrm{m}$ length for hyphae, compared with $<1 \mu \mathrm{m}$ diameter for bacterial cells) suggests that fungal rDNA quantification underestimates fungal biomass in the microbiota. In the past five years, researchers have analyzed endogenous fungal communities in the oral cavity, gastrointestinal tract, skin, and mucosal sites in healthy and diseased individuals (168-172). In most studies, between 15 and 70 fungal genera have been identified, with Malassezia spp. predominant in the skin and Candida spp. predominant in the intestine. Recent work has defined mechanisms of Candida colonization resistance by intestinal anaerobic bacteria (173).

Our understanding of the reciprocal interplay between endogenous fungal communities and antifungal immunity remains limited. Despite high-quality studies that point to a central role for IL-17 in mucosal antifungal immunity, it remains unclear whether IL-17 controls the composition or diversity of commensal fungi. Administration of secukinumab, an IL-17-neutralizing Ab, in patients with Crohn's disease increased the rate of fungal infections, consistent with IL-17 directing mucosal antifungal immunity (174).

Recent work has examined dectin-1 in shaping endogenous fungal communities and mycobiota-triggered immune responses in the gut. $\mathrm{Clec}_{7 a^{-1}}$ mice developed intestinal inflammation and Candida and Trichosporon spp. overgrowth in the gut (168). In turn, $\mathrm{Clec}^{-a^{--}}$mice were unable to control Candida during dextran sulfate sodium-induced colitis and benefited from fluconazole therapy. In the absence of commensal Candida, $C l e c 7 a^{-/-}$mice were more resistant to colitis and exhibited a reduction in colonic antimicrobial 
peptides that target Gram-positive bacteria, leading to an increase in commensal Lactobacillus murinus bacteria, Treg expansion, and resistance to colitis (175). Colonization of $C l e c 7 a^{-1-}$ mice with $C$. tropicalis reversed these effects and promoted intestinal inflammation. These findings suggest that dectin- 1 signaling indirectly influences the intestinal bacterial microbiota. In humans, a two-marker CLEC7A haplotype is associated with treatment-refractory ulcerative colitis (168). Another study identified a negative correlation between a CLEC7A single nucleotide polymorphism (SNP) and Malassezia sympodialis abundance in the gut of inflammatory bowel disease (IBD) patients, though no additional (positive or negative) correlations with other fungi were reported, likely due to the small cohort size (176). These studies highlight a protective role for dectin-1 in intestinal mycobiota control and antifungal immunity.

Dectin-3 was recently reported to contribute to control of intestinal Candida and colitis. Intestinal $C$. tropicalis overgrowth in Clec $4 d^{-1}$ mice was accompanied by a decrease in Th17 cells, impaired macrophage fungal phagocytosis, and defective intestinal epithelial cell barrier function (177). Consistent with these findings, antifungal treatment of $\mathrm{Clec} \mathrm{d}^{-1-}$ mice reduced $C$. tropicalis burden and ameliorated colitis.

CARD9 has also been implicated in IBD pathogenesis, as a nonsynonymous SNP in the CARD9-coding region strongly associates with IBD risk (178). In experimental colitis models, CARD9 signaling can be protective against fungi that contact the intestinal mucosa during colitis. Furthermore, antifungal drugs partially ameliorate intestinal inflammation in this context (179). Analysis of the intestinal microbiota of Card $9^{-1-}$ mice revealed alteration in fungal and bacterial communities compared with WT animals. The

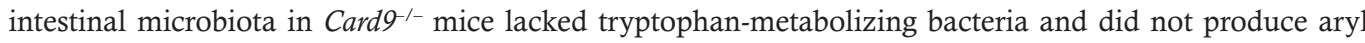
hydrocarbon receptor ligands (180). As a result, $\operatorname{Card}^{9^{--}}$mice showed defective expression of $I l 22$ and antimicrobial peptide-encoding genes Reg3g and Reg3b. Altogether, these findings suggest CARD9 may dually control fungal and bacterial populations in the gut.

Immunologic effects of intestinal fungi extend beyond the gut. In a house dust mite-induced airway allergy model, disruption of the gut fungal community with antifungal drugs increased disease severity (181). Aspergillus amstelodami, Epicoccum nigrum, and Wallemia sebi increased in abundance during antifungal drug-induced fungal dysbiosis, and intestinal supplementation with these strains replicated the detrimental effects of antifungal drugs on lung allergy (181). Similarly, antibiotic-induced fungal overgrowth exacerbated papain-induced lung allergy by promoting intestinal Candida overgrowth (182). This phenomenon, first demonstrated with $C$. albicans (183), is recapitulated by multiple Candida species and is mediated in part by eicosanoids (182). Thus, there is a complex interplay between endogenous fungal communities and innate immune tone and responses, both at local and distant sites.

\section{Conclusions and future perspectives}

The field of antifungal immunity has rapidly advanced in the past decade, a period marked by the dissection of fungus-specific innate and adaptive immune responses and convergence of human clinical and animal model data. The advances during this era are exemplified by insights into the pivotal role of dectin-1/ CARD9 and IL-17 pathways in antifungal immunity. The discovery of $\beta$-glucan-induced trained immunity and conserved sterilizing immunity-mediating epitopes lays the foundation for clinical trials to test vaccine protection against multiple fungal genera and species. Important areas of future research include elucidation of the role of epithelial surfaces in fungal virulence and antifungal defense and the intercellular crosstalk underlying innate and adaptive antifungal immunity. It is likely that additional fungal recognition receptors and response pathways remain to be discovered, both in animal models and in human genetic studies. How the composition, diversity, and metabolism of endogenous fungal communities contribute to immune homeostasis and to inflammatory disorders remains largely unexplored, yet are central for deciphering the contribution of fungi to diverse states of human health and disease.

\section{Acknowledgments}

The authors receive support from the Division of Intramural Research of the National Institute of Allergy and Infectious Diseases (MSL), NIH grants AI093808 (TMH), DK098310 (IDI), AI123819 (IDI), a Kenneth Rainin Foundation Award (IDI), a Burroughs Wellcome Fund Investigator in the Pathogenesis of Infectious Diseases Award (TMH), and a Geoffrey Beene Foundation Award (TMH). NIH Core Grant P30 CA008748 to MSKCC provided support for this manuscript. The authors apologize to many contributors in the field whose work could not be cited due to space limitations. 
Address correspondence to: Michail S. Lionakis, National Institutes of Health, 9000 Rockville Pike, Building 10, Room 11C102, Bethesda, Maryland, 20892, USA. Phone: 301.443.5089; E-mail: lionakism@ niaid.nih.gov. Or to: Iliyan D. Iliev, Weill Cornell Medicine, Belfer Research Building, Room BB 718, 413 East 69th Street, New York, New York 10021, USA. Phone: 646.962.7236; E-mail: iliev@med.cornell. edu. Or to: Tobias M. Hohl, Memorial Sloan Kettering Cancer Center, 1275 York Avenue, Box 9, New York, New York, 10065, USA. Phone: 646.888.3596; E-mail: hohlt@mskcc.org.

1. Brown GD, Denning DW, Gow NA, Levitz SM, Netea MG, White TC. Hidden killers: human fungal infections. Sci Transl Med. 2012;4(165):165rv13.

2. Saville SP, Lazzell AL, Monteagudo C, Lopez-Ribot JL. Engineered control of cell morphology in vivo reveals distinct roles for yeast and filamentous forms of Candida albicans during infection. Eukaryotic Cell. 2003;2(5):1053-1060.

3. Grzybowski A, Pietrzak K. Robert Remak (1815-1865): discoverer of the fungal character of dermatophytoses. Clin Dermatol. 2013;31(6):802-805.

4. Fisher MC, et al. Emerging fungal threats to animal, plant and ecosystem health. Nature. 2012;484(7393):186-194.

5. Underhill DM, Pearlman E. Immune interactions with pathogenic and commensal fungi: a two-way street. Immunity. 2015;43(5):845-858.

6. Rick EM, Woolnough K, Pashley CH, Wardlaw AJ. Allergic fungal airway disease. J Investig Allergol Clin Immunol. 2016;26(6):344-354.

7. Wu F, Groopman JD, Pestka JJ. Public health impacts of foodborne mycotoxins. Annu Rev Food Sci Technol. 2014;5:351-372.

8. Hohl TM. Overview of vertebrate animal models of fungal infection. J Immunol Methods. 2014;410:100-112.

9. Erwig LP, Gow NA. Interactions of fungal pathogens with phagocytes. Nat Rev Microbiol. 2016;14(3):163-176.

10. Rappleye CA, Eissenberg LG, Goldman WE. Histoplasma capsulatum alpha-(1,3)-glucan blocks innate immune recognition by the beta-glucan receptor. Proc Natl Acad Sci USA. 2007;104(4):1366-1370.

11. Garfoot AL, Shen Q, Wüthrich M, Klein BS, Rappleye CA. The Eng1 $\beta$-glucanase enhances histoplasma virulence by reducing B-glucan exposure. MBio. 2016;7(2):e01388-e01315.

12. Aimanianda $\mathrm{V}$, et al. Surface hydrophobin prevents immune recognition of airborne fungal spores. Nature. 2009;460(7259):1117-1121.

13. Fontaine T, et al. Galactosaminogalactan, a new immunosuppressive polysaccharide of Aspergillus fumigatus. PLoS Pathog. 2011;7(11):e1002372.

14. Gravelat FN, et al. Aspergillus galactosaminogalactan mediates adherence to host constituents and conceals hyphal $\beta$-glucan from the immune system. PLoS Pathog. 2013;9(8):e1003575.

15. Netea MG, et al. Immune sensing of Candida albicans requires cooperative recognition of mannans and glucans by lectin and Toll-like receptors. J Clin Invest. 2006;116(6):1642-1650.

16. Doering TL. How sweet it is! Cell wall biogenesis and polysaccharide capsule formation in Cryptococcus neoformans. Annu Rev Microbiol. 2009;63:223-247.

17. Lee MJ, Sheppard DC. Recent advances in the understanding of the Aspergillus fumigatus cell wall. J Microbiol. 2016;54(3):232242.

18. Netea MG, Brown GD, Kullberg BJ, Gow NA. An integrated model of the recognition of Candida albicans by the innate immune system. Nat Rev Microbiol. 2008;6(1):67-78.

19. Garlanda C, et al. Non-redundant role of the long pentraxin PTX3 in anti-fungal innate immune response. Nature. 2002;420(6912):182-186.

20. Moalli F, et al. Role of complement and Fc \{gamma $\}$ receptors in the protective activity of the long pentraxin PTX3 against Aspergillus fumigatus. Blood. 2010;116(24):5170-5180.

21. Cunha C, et al. Genetic PTX3 deficiency and aspergillosis in stem-cell transplantation. N Engl J Med. 2014;370(5):421-432.

22. Taylor PR, et al. Dectin-1 is required for beta-glucan recognition and control of fungal infection. Nat Immunol. 2007;8(1):31-38.

23. Saijo S, et al. Dectin-1 is required for host defense against Pneumocystis carinii but not against Candida albicans. Nat Immunol. 2007;8(1):39-46.

24. Werner JL, et al. Requisite role for the dectin-1 beta-glucan receptor in pulmonary defense against Aspergillus fumigatus. $J$ Immunol. 2009;182(8):4938-4946.

25. Goodridge HS, et al. Activation of the innate immune receptor Dectin-1 upon formation of a 'phagocytic synapse'. Nature. 2011;472(7344):471-475.

26. Deng Z, et al. Tyrosine phosphatase SHP-2 mediates C-type lectin receptor-induced activation of the kinase Syk and anti-fungal TH17 responses. Nat Immunol. 2015;16(6):642-652.

27. Strasser D, et al. Syk kinase-coupled C-type lectin receptors engage protein kinase $C$ - $\sigma$ to elicit Card 9 adaptor-mediated innate immunity. Immunity. 2012;36(1):32-42.

28. Roth S, et al. Vav proteins are key regulators of Card9 signaling for innate antifungal immunity. Cell Rep. 2016;17(10):2572-2583.

29. Gross O, et al. Card9 controls a non-TLR signalling pathway for innate anti-fungal immunity. Nature. 2006;442(7103):651-656.

30. Rogers NC, et al. Syk-dependent cytokine induction by Dectin-1 reveals a novel pattern recognition pathway for C type lectins. Immunity. 2005;22(4):507-517.

31. Gringhuis SI, et al. Dectin-1 directs T helper cell differentiation by controlling noncanonical NF-kappaB activation through Raf-1 and Syk. Nat Immunol. 2009;10(2):203-213.

32. Gross $\mathrm{O}$, et al. Syk kinase signalling couples to the Nlrp3 inflammasome for anti-fungal host defence. Nature. 2009;459(7245):433-436.

33. Gringhuis SI, et al. Dectin-1 is an extracellular pathogen sensor for the induction and processing of IL-1 $\beta$ via a noncanonical caspase-8 inflammasome. Nat Immunol. 2012;13(3):246-254. 
34. Karki R, et al. Concerted activation of the AIM2 and NLRP3 inflammasomes orchestrates host protection against Aspergillus infection. Cell Host Microbe. 2015;17(3):357-368.

35. Yang CS, et al. The autophagy regulator Rubicon is a feedback inhibitor of CARD9-mediated host innate immunity. Cell Host Microbe. 2012;11(3):277-289.

36. LeibundGut-Landmann S, et al. Syk- and CARD9-dependent coupling of innate immunity to the induction of T helper cells that produce interleukin 17. Nat Immunol. 2007;8(6):630-638.

37. del Fresno $C$, et al. Interferon- $\beta$ production via Dectin-1-Syk-IRF5 signaling in dendritic cells is crucial for immunity to $C$. albicans. Immunity. 2013;38(6):1176-1186.

38. Majer $\mathrm{O}$, et al. Type I interferons promote fatal immunopathology by regulating inflammatory monocytes and neutrophils during Candida infections. PLoS Pathog. 2012;8(7):e1002811.

39. Jia XM, et al. CARD9 mediates Dectin-1-induced ERK activation by linking Ras-GRF1 to H-Ras for antifungal immunity. J Exp Med. 2014;211(11):2307-2321.

40. Zhao X, et al. JNK1 negatively controls antifungal innate immunity by suppressing CD23 expression. Nat Med. 2017;23(3):337-346.

41. Strijbis K, et al. Bruton's tyrosine kinase (BTK) and Vav1 contribute to Dectin1-dependent phagocytosis of Candida albicans in macrophages. PLoS Pathog. 2013;9(6):e1003446.

42. Boyle KB, et al. Class IA phosphoinositide 3-kinase $\beta$ and $\delta$ regulate neutrophil oxidase activation in response to Aspergillus fumigatus hyphae. J Immunol. 2011;186(5):2978-2989.

43. Jhingran A, et al. Tracing conidial fate and measuring host cell antifungal activity using a reporter of microbial viability in the lung. Cell Rep. 2012;2(6):1762-1773.

44. Huang JH, et al. CR3 and Dectin-1 collaborate in macrophage cytokine response through association on lipid rafts and activation of Syk-JNK-AP-1 pathway. PLoS Pathog. 2015;11(7):e1004985.

45. Li X, et al. The $\beta$-glucan receptor Dectin-1 activates the integrin Mac-1 in neutrophils via Vav protein signaling to promote Candida albicans clearance. Cell Host Microbe. 2011;10(6):603-615.

46. Saijo S, et al. Dectin-2 recognition of alpha-mannans and induction of Th17 cell differentiation is essential for host defense against Candida albicans. Immunity. 2010;32(5):681-691.

47. Zhu LL, et al. C-type lectin receptors Dectin-3 and Dectin-2 form a heterodimeric pattern-recognition receptor for host defense against fungal infection. Immunity. 2013;39(2):324-334.

48. Ishikawa $\mathrm{T}$, et al. Identification of distinct ligands for the C-type lectin receptors Mincle and Dectin-2 in the pathogenic fungus Malassezia. Cell Host Microbe. 2013;13(4):477-488.

49. Wang H, et al. C-type lectin receptors differentially induce Th17 cells and vaccine immunity to the endemic mycosis of North America. J Immunol. 2014;192(3):1107-1119.

50. Wirnsberger G, et al. Inhibition of CBLB protects from lethal Candida albicans sepsis. Nat Med. 2016;22(8):915-923.

51. Xiao Y, et al. Targeting CBLB as a potential therapeutic approach for disseminated candidiasis. Nat Med. 2016;22(8):906-914.

52. Zhu LL, et al. E3 ubiquitin ligase Cbl-b negatively regulates C-type lectin receptor-mediated antifungal innate immunity. $J$ Exp Med. 2016;213(8):1555-1570.

53. Cao Z, et al. Ubiquitin ligase TRIM62 regulates CARD9-mediated anti-fungal immunity and intestinal inflammation. Immunity. 2015;43(4):715-726.

54. Sousa Mda G, et al. Restoration of pattern recognition receptor costimulation to treat chromoblastomycosis, a chronic fungal infection of the skin. Cell Host Microbe. 2011;9(5):436-443.

55. de Sousa Mda G, et al. Topical application of imiquimod as a treatment for chromoblastomycosis. Clin Infect Dis. 2014;58(12):1734-1737.

56. Wevers BA, et al. Fungal engagement of the C-type lectin mincle suppresses dectin-1-induced antifungal immunity. Cell Host Microbe. 2014;15(4):494-505.

57. Kottom TJ, et al. The interaction of pneumocystis with the C-type lectin receptor mincle exerts a significant role in host defense against infection. J Immunol. 2017;198(9):3515-3525.

58. Wells CA, et al. The macrophage-inducible C-type lectin, mincle, is an essential component of the innate immune response to Candida albicans. J Immunol. 2008;180(11):7404-7413

59. Marakalala MJ, et al. Differential adaptation of Candida albicans in vivo modulates immune recognition by dectin-1. PLoS Pathog. 2013;9(4):e1003315.

60. Whitney PG, et al. Syk signaling in dendritic cells orchestrates innate resistance to systemic fungal infection. PLoS Pathog 2014;10(7):e1004276.

61. Bär E, Whitney PG, Moor K, Reis e Sousa C, LeibundGut-Landmann S. IL-17 regulates systemic fungal immunity by controlling the functional competence of NK cells. Immunity. 2014;40(1):117-127.

62. Bozza S, et al. PTX3 binds MD-2 and promotes TRIF-dependent immune protection in aspergillosis. J Immunol. 2014;193(5):2340-2348

63. Herbst S, et al. Phagocytosis-dependent activation of a TLR9-BTK-calcineurin-NFAT pathway co-ordinates innate immunity to Aspergillus fumigatus. EMBO Mol Med. 2015;7(3):240-258.

64. Lanternier F, et al. Primary immunodeficiencies underlying fungal infections. Curr Opin Pediatr. 2013;25(6):736-747.

65. Lionakis MS, Netea MG, Holland SM. Mendelian genetics of human susceptibility to fungal infection. Cold Spring Harb Perspect Med. 2014;4(6):a019638.

66. Warnatsch A, et al. Reactive oxygen species localization programs inflammation to clear microbes of different size. Immunity. 2017;46(3):421-432.

67. Akoumianaki T, et al. Aspergillus cell wall melanin blocks LC3-associated phagocytosis to promote pathogenicity. Cell Host Microbe. 2016;19(1):79-90.

68. Oikonomou V, et al. Noncanonical fungal autophagy inhibits inflammation in response to IFN- $\gamma$ via DAPK1. Cell Host Microbe 2016;20(6):744-757

69. Subramanian Vignesh K, Landero Figueroa JA, Porollo A, Caruso JA, Deepe GS. Granulocyte macrophage-colony stimulat- 
ing factor induced $\mathrm{Zn}$ sequestration enhances macrophage superoxide and limits intracellular pathogen survival. Immunity. 2013;39(4):697-710.

70. Leal SM, et al. Targeting iron acquisition blocks infection with the fungal pathogens Aspergillus fumigatus and Fusarium oxysporum. PLoS Pathog. 2013;9(7):e1003436.

71. Zarember KA, Sugui JA, Chang YC, Kwon-Chung KJ, Gallin JI. Human polymorphonuclear leukocytes inhibit Aspergillus fumigatus conidial growth by lactoferrin-mediated iron depletion. J Immunol. 2007;178(10):6367-6373.

72. Gazendam RP, et al. Two independent killing mechanisms of Candida albicans by human neutrophils: evidence from innate immunity defects. Blood. 2014;124(4):590-597.

73. Gazendam RP, et al. Human neutrophils use different mechanisms to kill Aspergillus fumigatus conidia and hyphae: evidence from phagocyte defects. J Immunol. 2016;196(3):1272-1283.

74. Branzk N, et al. Neutrophils sense microbe size and selectively release neutrophil extracellular traps in response to large pathogens. Nat Immunol. 2014;15(11):1017-1025.

75. Jaillon S, et al. The humoral pattern recognition receptor PTX3 is stored in neutrophil granules and localizes in extracellular traps. J Exp Med. 2007;204(4):793-804.

76. Urban CF, et al. Neutrophil extracellular traps contain calprotectin, a cytosolic protein complex involved in host defense against Candida albicans. PLoS Pathog. 2009;5(10):e1000639.

77. Clark HL, et al. Zinc and manganese chelation by neutrophil S100A8/A9 (calprotectin) limits extracellular Aspergillus fumigatus hyphal growth and corneal infection. J Immunol. 2016;196(1):336-344.

78. Bruns S, et al. Production of extracellular traps against Aspergillus fumigatus in vitro and in infected lung tissue is dependent on invading neutrophils and influenced by hydrophobin RodA. PLoS Pathog. 2010;6(4):e1000873.

79. Lee MJ, et al. The fungal exopolysaccharide galactosaminogalactan mediates virulence by enhancing resistance to neutrophil extracellular traps. PLoS Pathog. 2015;11(10):e1005187.

80. Gladiator A, Wangler N, Trautwein-Weidner K, LeibundGut-Landmann S. Cutting edge: IL-17-secreting innate lymphoid cells are essential for host defense against fungal infection. J Immunol. 2013;190(2):521-525.

81. Conti HR, et al. Oral-resident natural Th17 cells and $\gamma \delta \mathrm{T}$ cells control opportunistic Candida albicans infections. $J$ Exp Med. 2014;211(10):2075-2084.

82. Kashem SW, Riedl MS, Yao C, Honda CN, Vulchanova L, Kaplan DH. Nociceptive sensory fibers drive interleukin-23 production from $\mathrm{CD} 31 \mathrm{~b}^{+}$dermal dendritic cells and drive protective cutaneous immunity. Immunity. 2015;43(3):515-526.

83. Conti HR, et al. IL-17 receptor signaling in oral epithelial cells is critical for protection against oropharyngeal candidiasis. Cell Host Microbe. 2016;20(5):606-617.

84. Ramani K, et al. The kallikrein-kinin system: a novel mediator of IL-17-driven anti-Candida immunity in the kidney. PLoS Pathog. 2016;12(11):e1005952.

85. Huppler AR, Conti HR, Hernández-Santos N, Darville T, Biswas PS, Gaffen SL. Role of neutrophils in IL-17-dependent immunity to mucosal candidiasis. J Immunol. 2014;192(4):1745-1752.

86. Trautwein-Weidner K, Gladiator A, Nur S, Diethelm P, LeibundGut-Landmann S. IL-17-mediated antifungal defense in the oral mucosa is independent of neutrophils. Mucosal Immunol. 2015;8(2):221-231.

87. Altmeier S, Toska A, Sparber F, Teijeira A, Halin C, LeibundGut-Landmann S. IL-1 coordinates the neutrophil response to C. albicans in the oral mucosa. PLoS Pathog. 2016;12(9):e1005882.

88. Liu M, et al. The endothelial cell receptor GRP78 is required for mucormycosis pathogenesis in diabetic mice. J Clin Invest. 2010;120(6):1914-1924.

89. Gebremariam T, et al. Bicarbonate correction of ketoacidosis alters host-pathogen interactions and alleviates mucormycosis. J Clin Invest. 2016;126(6):2280-2294.

90. Gebremariam T, et al. CotH3 mediates fungal invasion of host cells during mucormycosis. J Clin Invest. 2014;124(1):237-250.

91. Phan QT, et al. Als3 is a Candida albicans invasin that binds to cadherins and induces endocytosis by host cells. PLoS Biol. 2007;5(3):e64

92. Modrzewska B, Kurnatowski P. Adherence of Candida sp. to host tissues and cells as one of its pathogenicity features. Ann Parasitol. 2015;61(1):3-9.

93. Schmidt CS, et al. NDV-3, a recombinant alum-adjuvanted vaccine for Candida and Staphylococcus aureus, is safe and immunogenic in healthy adults. Vaccine. 2012;30(52):7594-7600.

94. Moyes DL, et al. Candidalysin is a fungal peptide toxin critical for mucosal infection. Nature. 2016;532(7597):64-68

95. Kerr SC, et al. FleA expression in Aspergillus fumigatus is recognized by fucosylated structures on mucins and macrophages to prevent lung infection. PLoS Pathog. 2016;12(4):e1005555.

96. Liu H, et al. Aspergillus fumigatus CalA binds to integrin $\alpha 5 \beta 1$ and mediates host cell invasion. Nat Microbiol. 2016;2:16211

97. Taylor PR, et al. Activation of neutrophils by autocrine IL-17A-IL-17RC interactions during fungal infection is regulated by IL-6, IL-23, ROR $\gamma$ and dectin-2. Nat Immunol. 2014;15(2):143-151.

98. Savers A, et al. Infection-mediated priming of phagocytes protects against lethal secondary Aspergillus fumigatus challenge. PLoS One. 2016;11(4):e0153829.

99. Ngo LY, Kasahara S, Kumasaka DK, Knoblaugh SE, Jhingran A, Hohl TM. Inflammatory monocytes mediate early and organ specific innate defense during systemic candidiasis. J Infect Dis. 2014;209(1):109-119.

100. Espinosa V, et al. Inflammatory monocytes orchestrate innate antifungal immunity in the lung. PLoS Pathog. 2014;10(2):e1003940.

101. Sterkel AK, et al. Fungal mimicry of a mammalian aminopeptidase disables innate immunity and promotes pathogenicity. Cell Host Microbe. 2016;19(3):361-374.

102. Quintin J, et al. Candida albicans infection affords protection against reinfection via functional reprogramming of monocytes. Cell Host Microbe. 2012;12(2):223-232.

103. Cheng SC, et al. mTOR- and HIF-1 $\alpha$-mediated aerobic glycolysis as metabolic basis for trained immunity. Science. 2014;345(6204):1250684.

104. Arts RJ, et al. Immunometabolic pathways in BCG-induced trained immunity. Cell Rep. 2016;17(10):2562-2571. 
105. Novakovic B, et al. $\beta$-Glucan reverses the epigenetic state of LPS-induced immunological tolerance. Cell. 2016;167(5):1354-1368.e14.

106. Ifrim DC, et al. Defective trained immunity in patients with STAT-1-dependent chronic mucocutaneaous candidiasis. Clin Exp Immunol. 2015;181(3):434-440.

107. Casadevall A, Pirofski LA. Immunoglobulins in defense, pathogenesis, and therapy of fungal diseases. Cell Host Microbe. 2012;11(5):447-456.

108. Rapaka RR, et al. Conserved natural IgM antibodies mediate innate and adaptive immunity against the opportunistic fungus Pneumocystis murina. J Exp Med. 2010;207(13):2907-2919.

109. Szymczak WA, Davis MJ, Lundy SK, Dufaud C, Olszewski M, Pirofski LA. X-linked immunodeficient mice exhibit enhanced susceptibility to Cryptococcus neoformans Infection. MBio. 2013;4(4):e00265.

110. Torosantucci A, et al. A novel glyco-conjugate vaccine against fungal pathogens. J Exp Med. 2005;202(5):597-606.

111. Torosantucci A, et al. Protection by anti-beta-glucan antibodies is associated with restricted beta-1,3 glucan binding specificity and inhibition of fungal growth and adherence. PLoS One. 2009;4(4):e5392.

112. Wharton RE, Stefanov EK, King RG, Kearney JF. Antibodies generated against Streptococci protect in a mouse model of disseminated aspergillosis. J Immunol. 2015;194(9):4387-4396.

113. Sandini S, La Valle R, Deaglio S, Malavasi F, Cassone A, De Bernardis F. A highly immunogenic recombinant and truncated protein of the secreted aspartic proteases family (rSap2t) of Candida albicans as a mucosal anticandidal vaccine. FEMS Immunol Med Microbiol. 2011;62(2):215-224.

114. Rivera $\mathrm{A}$, et al. Innate immune activation and $\mathrm{CD} 4^{+} \mathrm{T}$ cell priming during respiratory fungal infection. Immunity. 2006;25(4):665-675.

115. Wüthrich M, et al. A TCR transgenic mouse reactive with multiple systemic dimorphic fungi. J Immunol. 2011;187(3):1421-1431.

116. Trautwein-Weidner K, et al. Antigen-specific Th17 cells are primed by distinct and complementary dendritic cell subsets in oropharyngeal candidiasis. PLoS Pathog. 2015;11(10):e1005164.

117. Bär E, et al. A novel Th cell epitope of Candida albicans mediates protection from fungal infection. J Immunol. 2012;188(11):5636-5643.

118. Wüthrich M, et al. Calnexin induces expansion of antigen-specific CD4(+) T cells that confer immunity to fungal ascomycetes via conserved epitopes. Cell Host Microbe. 2015;17(4):452-465.

119. Wiesner DL, et al. Chitin recognition via chitotriosidase promotes pathologic type-2 helper $\mathrm{T}$ cell responses to cryptococcal infection. PLoS Pathog. 2015;11(3):e1004701.

120. Verma A, Wüthrich M, Deepe G, Klein B. Adaptive immunity to fungi. Cold Spring Harb Perspect Med. 2014;5(3):a019612.

121. Hohl TM, et al. Inflammatory monocytes facilitate adaptive CD4 T cell responses during respiratory fungal infection. Cell Host Microbe. 2009;6(5):470-481.

122. Ersland K, Wüthrich M, Klein BS. Dynamic interplay among monocyte-derived, dermal, and resident lymph node dendritic cells during the generation of vaccine immunity to fungi. Cell Host Microbe. 2010;7(6):474-487.

123. Wüthrich M, Ersland K, Sullivan T, Galles K, Klein BS. Fungi subvert vaccine T cell priming at the respiratory mucosa by preventing chemokine-induced influx of inflammatory monocytes. Immunity. 2012;36(4):680-692.

124. Rivera A, et al. Dectin-1 diversifies Aspergillus fumigatus-specific T cell responses by inhibiting T helper type $1 \mathrm{CD} 4 \mathrm{~T}$ cell differentiation. J Exp Med. 2011;208(2):369-381.

125. Schlitzer A, et al. IRF4 transcription factor-dependent CD11 $\mathrm{b}^{+}$dendritic cells in human and mouse control mucosal IL-17 cytokine responses. Immunity. 2013;38(5):970-983.

126. Kashem SW, et al. Candida albicans morphology and dendritic cell subsets determine $\mathrm{T}$ helper cell differentiation. Immunity. 2015;42(2):356-366.

127. Wüthrich M, et al. Vaccine-induced protection against 3 systemic mycoses endemic to North America requires Th17 cells in mice. J Clin Invest. 2011;121(2):554-568.

128. Hung CY, Gonzalez A, Wüthrich M, Klein BS, Cole GT. Vaccine immunity to coccidioidomycosis occurs by early activation of three signal pathways of Thelper cell response (Th1, Th2, and Th17). Infect Immun. 2011;79(11):4511-4522.

129. Pandiyan $\mathrm{P}$, et al. CD4(+)CD25(+)Foxp3(+) regulatory T cells promote Th17 cells in vitro and enhance host resistance in mouse Candida albicans Th17 cell infection model. Immunity. 2011;34(3):422-434.

130. Whibley N, et al. Expansion of Foxp3(+) T-cell populations by Candida albicans enhances both Th17-cell responses and fungal dissemination after intravenous challenge. Eur J Immunol. 2014;44(4):1069-1083.

131. Lionakis MS, et al. Chemokine receptor Ccr1 drives neutrophil-mediated kidney immunopathology and mortality in invasive candidiasis. PLoS Pathog. 2012;8(8):e1002865.

132. Upadhya R, Lam WC, Maybruck B, Specht CA, Levitz SM, Lodge JK. Induction of protective immunity to cryptococcal infection in mice by a heat-killed, chitosan-deficient strain of Cryptococcus neoformans. MBio. 2016;7(3):e00547.

133. O'Dea EM, et al. Eosinophils are recruited in response to chitin exposure and enhance Th2-mediated immune pathology in Aspergillus fumigatus infection. Infect Immun. 2014;82(8):3199-3205.

134. Lilly LM, Scopel M, Nelson MP, Burg AR, Dunaway CW, Steele C. Eosinophil deficiency compromises lung defense against Aspergillus fumigatus. Infect Immun. 2014;82(3):1315-1325.

135. Guerra ES, et al. Central role of IL-23 and IL-17 producing eosinophils as immunomodulatory effector cells in acute pulmonary aspergillosis and allergic asthma. PLoS Pathog. 2017;13(1):e1006175.

136. Eddens T, et al. Eosinophils contribute to early clearance of Pneumocystis murina infection. J Immunol. 2015;195(1):185-193.

137. Segal BH, Leto TL, Gallin JI, Malech HL, Holland SM. Genetic, biochemical, and clinical features of chronic granulomatous disease. Medicine (Baltimore). 2000;79(3):170-200.

138. Nauseef WM, Root RK, Malech HL. Biochemical and immunologic analysis of hereditary myeloperoxidase deficiency. J Clin Invest. 1983;71(5):1297-1307.

139. Greenblatt MB, Aliprantis A, Hu B, Glimcher LH. Calcineurin regulates innate antifungal immunity in neutrophils. J Exp Med. 2010;207(5):923-931. 
140. Henriet S, Verweij PE, Holland SM, Warris A. Invasive fungal infections in patients with chronic granulomatous disease. $A d v$ Exp Med Biol. 2013;764:27-55.

141. Reeves EP, et al. Killing activity of neutrophils is mediated through activation of proteases by $\mathrm{K}^{+}$flux. Nature. 2002;416(6878):291-297.

142. Bax HI, et al. Interferon alpha treatment of patients with impaired interferon gamma signaling. J Clin Immunol. 2013;33(5):991-1001

143. Browne SK, et al. Adult-onset immunodeficiency in Thailand and Taiwan. N Engl J Med. 2012;367(8):725-734.

144. Rosen LB, et al. Anti-GM-CSF autoantibodies in patients with cryptococcal meningitis. J Immunol. 2013;190(8):3959-3966.

145. Punatar AD, Kusne S, Blair JE, Seville MT, Vikram HR. Opportunistic infections in patients with pulmonary alveolar proteinosis. J Infect. 2012;65(2):173-179.

146. Kasahara S, Jhingran A, Dhingra S, Salem A, Cramer RA, Hohl TM. Role of granulocyte-macrophage colony-stimulating factor signaling in regulating neutrophil antifungal activity and the oxidative burst during respiratory fungal challenge. J Infect Dis. 2016;213(8):1289-1298.

147. O'Shea JJ, Holland SM, Staudt LM. JAKs and STATs in immunity, immunodeficiency, and cancer. N Engl J Med. 2013;368(2):161-170.

148. Toubiana J, et al. Heterozygous STAT1 gain-of-function mutations underlie an unexpectedly broad clinical phenotype. Blood. 2016;127(25):3154-3164.

149. Odio CD, et al. Endemic mycoses in patients with STAT3-mutated hyper-IgE (Job) syndrome. J Allergy Clin Immunol. 2015;136(5):1411-3.e1.

150.Spinner MA, et al. GATA2 deficiency: a protean disorder of hematopoiesis, lymphatics, and immunity. Blood. 2014;123(6):809-821.

151.Ling Y, et al. Inherited IL-17RC deficiency in patients with chronic mucocutaneous candidiasis. J Exp Med. 2015;212(5):619-631.

152. Puel A, et al. Chronic mucocutaneous candidiasis in humans with inborn errors of interleukin-17 immunity. Science. 2011;332(6025):65-68

153. Ferre EM, et al. Redefined clinical features and diagnostic criteria in autoimmune polyendocrinopathy-candidiasis-ectodermal dystrophy. JCI Insight. 2016;1(13):e88782.

154. Shearer WT, et al. Establishing diagnostic criteria for severe combined immunodeficiency disease (SCID), leaky SCID, and Omenn syndrome: the Primary Immune Deficiency Treatment Consortium experience. J Allergy Clin Immunol. 2014;133(4):1092-1098.

155. Okada S, et al. IMMUNODEFICIENCIES. Impairment of immunity to Candida and Mycobacterium in humans with biallelic RORC mutations. Science. 2015;349(6248):606-613.

156. Cunha C, et al. Dectin-1 Y238X polymorphism associates with susceptibility to invasive aspergillosis in hematopoietic transplantation through impairment of both recipient- and donor-dependent mechanisms of antifungal immunity. Blood 2010;116(24):5394-5402.

157. Drummond RA, Lionakis MS. Mechanistic insights into the role of C-type lectin receptor/CARD9 signaling in human antifungal immunity. Front Cell Infect Microbiol. 2016;6:39.

158. Lanternier F, et al. Inherited CARD9 deficiency in otherwise healthy children and adults with Candida species-induced meningoencephalitis, colitis, or both. J Allergy Clin Immunol. 2015;135(6):1558-68.e2.

159. Drummond RA, et al. CARD9-dependent neutrophil recruitment protects against fungal invasion of the central nervous system. PLoS Pathog. 2015;11(12):e1005293.

160. Drewniak A, et al. Invasive fungal infection and impaired neutrophil killing in human CARD9 deficiency. Blood. 2013;121(13):2385-2392.

161. Jhingran A, et al. Compartment-specific and sequential role of MyD88 and CARD9 in chemokine induction and innate defense during respiratory fungal infection. PLoS Pathog. 2015;11(1):e1004589.

162. Rieber N, et al. Extrapulmonary Aspergillus infection in patients with CARD9 deficiency. JCI Insight. 2016;1(17):e89890.

163. Caffrey AK, et al. IL-1 $\alpha$ signaling is critical for leukocyte recruitment after pulmonary Aspergillus fumigatus challenge. $P L o S$ Pathog. 2015;11(1):e1004625.

164. Gavino C, et al. CARD9 deficiency and spontaneous central nervous system candidiasis: complete clinical remission with GMCSF therapy. Clin Infect Dis. 2014;59(1):81-84.

165. Gavino C, et al. Impaired RASGRF1/ERK-mediated GM-CSF response characterizes CARD9 deficiency in French-Canadians. J Allergy Clin Immunol. 2016;137(4):1178-1188.e1.

166. Tang J, Iliev ID, Brown J, Underhill DM, Funari VA. Mycobiome: approaches to analysis of intestinal fungi. J Immunol Methods. 2015;421:112-121.

167. Underhill DM, Iliev ID. The mycobiota: interactions between commensal fungi and the host immune system. Nat Rev Immunol. 2014;14(6):405-416

168. Iliev ID, et al. Interactions between commensal fungi and the C-type lectin receptor Dectin-1 influence colitis. Science. 2012;336(6086):1314-1317.

169. Findley K, et al. Topographic diversity of fungal and bacterial communities in human skin. Nature. 2013;498(7454):367-370.

170. Dupuy AK, et al. Redefining the human oral mycobiome with improved practices in amplicon-based taxonomy: discovery of Malassezia as a prominent commensal. PLoS ONE. 2014;9(3):e90899.

171. Frykman PK, et al. Characterization of bacterial and fungal microbiome in children with Hirschsprung disease with and without a history of enterocolitis: a multicenter study. PLoS ONE. 2015;10(4):e0124172.

172. Nguyen LD, Viscogliosi E, Delhaes L. The lung mycobiome: an emerging field of the human respiratory microbiome. Front Microbiol. 2015;6:89.

173. Fan D, et al. Activation of HIF-1 $\alpha$ and LL-37 by commensal bacteria inhibits Candida albicans colonization. Nat Med. 2015;21(7):808-814

174. Hueber W, et al. Secukinumab, a human anti-IL-17A monoclonal antibody, for moderate to severe Crohn's disease: unexpected results of a randomised, double-blind placebo-controlled trial. Gut. 2012;61(12):1693-1700.

175. Tang C, et al. Inhibition of Dectin-1 signaling ameliorates colitis by inducing Lactobacillus-mediated regulatory $\mathrm{T}$ cell expan- 
sion in the intestine. Cell Host Microbe. 2015;18(2):183-197.

176. Sokol H, et al. Fungal microbiota dysbiosis in IBD [published online ahead of print February 3, 2016]. Gut. https://doi.org/ 10.1136/gutjnl-2015-310746.

177. Wang T, et al. Dectin-3 deficiency promotes colitis development due to impaired antifungal innate immune responses in the gut. PLoS Pathog. 2016;12(6):e1005662.

178. Khor B, Gardet A, Xavier RJ. Genetics and pathogenesis of inflammatory bowel disease. Nature. 2011;474(7351):307-317.

179. Sokol H, et al. Card9 mediates intestinal epithelial cell restitution, T-helper 17 responses, and control of bacterial infection in mice. Gastroenterology. 2013;145(3):591-601.e3.

180. Lamas B, et al. CARD9 impacts colitis by altering gut microbiota metabolism of tryptophan into aryl hydrocarbon receptor ligands. Nat Med. 2016;22(6):598-605.

181. Wheeler ML, et al. Immunological consequences of intestinal fungal dysbiosis. Cell Host Microbe. 2016;19(6):865-873.

182. Kim YG, Udayanga KG, Totsuka N, Weinberg JB, Núñez G, Shibuya A. Gut dysbiosis promotes M2 macrophage polarization and allergic airway inflammation via fungi-induced PGE $_{2}$. Cell Host Microbe. 2014;15(1):95-102.

183. Noverr MC, Noggle RM, Toews GB, Huffnagle GB. Role of antibiotics and fungal microbiota in driving pulmonary allergic responses. Infect Immun. 2004;72(9):4996-5003.

184. Loures FV, et al. Dectin-1 induces M1 macrophages and prominent expansion of CD8 $8^{+} \mathrm{IL}-17^{+}$cells in pulmonary Paracoccidioidomycosis. J Infect Dis. 2014;210(5):762-773.

185. Ifrim DC, et al. Role of Dectin-2 for host defense against systemic infection with Candida glabrata. Infect Immun. 2014;82(3):1064-1073.

186. Wang H, Li M, Lerksuthirat T, Klein B, Wüthrich M. The C-type lectin receptor mcl mediates vaccine-induced immunity against infection with Blastomyces dermatitidis. Infect Immun. 2015;84(3):635-642.

187. Serrano-Gómez D, Domínguez-Soto A, Ancochea J, Jimenez-Heffernan JA, Leal JA, Corbí AL. Dendritic cell-specific intercellular adhesion molecule 3-grabbing nonintegrin mediates binding and internalization of Aspergillus fumigatus conidia by dendritic cells and macrophages. J Immunol. 2004;173(9):5635-5643.

188. Lee SJ, Zheng NY, Clavijo M, Nussenzweig MC. Normal host defense during systemic candidiasis in mannose receptor-deficient mice. Infect Immun. 2003;71(1):437-445.

189. Netea MG, Van Der Graaf CA, Vonk AG, Verschueren I, Van Der Meer JW, Kullberg BJ. The role of Toll-like receptor (TLR) 2 and TLR4 in the host defense against disseminated candidiasis. J Infect Dis. 2002;185(10):1483-1489.

190. Yauch LE, Mansour MK, Shoham S, Rottman JB, Levitz SM. Involvement of CD14, Toll-like receptors 2 and 4, and MyD88 in the host response to the fungal pathogen Cryptococcus neoformans in vivo. Infect Immun. 2004;72(9):5373-5382.

191. Jouault T, et al. Candida albicans phospholipomannan is sensed through Toll-like receptors. J Infect Dis. 2003;188(1):165-172.

192. Figueiredo RT, et al. TLR4 recognizes Pseudallescheria boydii conidia and purified rhamnomannans. J Biol Chem. 2010;285(52):40714-40723.

193. Zhang Y, Wu J, Xin Z, Wu X. Aspergillus fumigatus triggers innate immune response via NOD1 signaling in human corneal epithelial cells. Exp Eye Res. 2014;127:170-178.

194. Wagener J, et al. Fungal chitin dampens inflammation through IL-10 induction mediated by NOD2 and TLR9 activation. PLoS Pathog. 2014;10(4):e1004050.

195. Tomalka J, et al. A novel role for the NLRC4 inflammasome in mucosal defenses against the fungal pathogen Candida albicans. PLoS Pathog. 2011;7(12):e1002379.

196. Hise AG, et al. An essential role for the NLRP3 inflammasome in host defense against the human fungal pathogen Candida albicans. Cell Host Microbe. 2009;5(5):487-497.

197. Tada H, et al. Saccharomyces cerevisiae- and Candida albicans-derived mannan induced production of tumor necrosis factor alpha by human monocytes in a CD14- and Toll-like receptor 4-dependent manner. Microbiol Immunol. 2002;46(7):503-512.

198. Means TK, et al. Evolutionarily conserved recognition and innate immunity to fungal pathogens by the scavenger receptors SCARF1 and CD36. J Exp Med. 2009;206(3):637-653.

199. Linden JR, De Paepe ME, Laforce-Nesbitt SS, Bliss JM. Galectin-3 plays an important role in protection against disseminated candidiasis. Med Mycol. 2013;51(6):641-651.

200. Hahn PY, Evans SE, Kottom TJ, Standing JE, Pagano RE, Limper AH. Pneumocystis carinii cell wall beta-glucan induces release of macrophage inflammatory protein-2 from alveolar epithelial cells via a lactosylceramide-mediated mechanism. $J$ Biol Chem. 2003;278(3):2043-2050. 